\title{
A POLISH TOPOLOGY FOR THE CLOSED SUBSETS OF A POLISH SPACE
}

\author{
GERALD BEER
}

(Communicated by Dennis Burke)

\begin{abstract}
Let $\langle X, d\rangle$ be a complete and separable metric space. The Wijsman topology on the nonempty closed subset $\operatorname{CL}(X)$ of $X$ is the weakest topology on $\mathrm{CL}(X)$ such that for each $x$ in $X$, the distance functional $A \rightarrow d(x, A)$ is continuous on $\operatorname{CL}(X)$. We show that this topology is Polish, and that the traditional extension of the topology to include the empty set among the closed sets is also Polish. We also compare the Borel class of a closed valued multifunction with its Borel class when viewed as a single-valued function into $\mathrm{CL}(X)$, equipped with Wijsman topology.
\end{abstract}

\section{INTRODUCTION}

Arguably, the most important class of metric spaces are the Polish spaces: metric spaces that are both completely metrizable and separable. They are basic objects in descriptive set theory and its applications, for example, the study of Borel structure, analytic sets, the projective hierarchy, measurable multifunctions and their measurable selections, and probability measures on metric spaces [Ch, CV, Hi, KR, KT, Ku, Pa, Wa]. It is the purpose of this article to show that the closed subsets of a Polish space may be equipped with a Polish topology that extends the initial topology on $X$ under the identification $x \rightarrow\{x\}$.

At this point, we introduce some notation and terminology. Let $\langle X, d\rangle$ be a metric space. For $A \subset X$ and $x \in X$, we denote $\inf \{d(x, a): a \in A\}$ by $d(x, A)$. For $x \in X$ and $r>0, S_{r}[x]$ will denote the open ball of radius $r$ with center $x$. A subset $A$ of $X$ is called $\varepsilon$-discrete provided $d\left(a_{1}, a_{2}\right)>\varepsilon$ for each pair of distinct points $\left\{a_{1}, a_{2}\right\}$ in $A$.

The set of nonempty closed subsets of $X$ will be denoted by $\operatorname{CL}(X)$, and the set of nonempty compact subsets of $X$ by $K(X)$. By a hyperspace topology, we simply mean a topology on $\mathrm{CL}(X)$. For each $A \in \mathrm{CL}(X)$ and $r>0$, we denote $\cup\left\{S_{r}[a]: a \in A\right\}$ by $S_{r}[A]$. Following [MN] we denote the continuous functions from $X$ to $R$ equipped with the topology of pointwise convergence

Received by the editors April 9, 1990.

1980 Mathematics Subject Classification (1985 Revision). Primary 54B20, 54E50; Secondary $54 \mathrm{C} 35,54 \mathrm{C} 60$.

Key words and phrases. Polish space, hyperspace, Wijsman topology, distance functional, topology of pointwise convergence, multifunction. 
by $C_{p}(X, R)$. Finally, if $T$ is a locally compact topological space, we denote by $T^{*}$ its Alexandroff one-point compactification (see, e.g., [En]).

\section{FAILURE OF STANDARD hyPERSPACE TOPOlOGIES to BE Polish}

In this section, $X$ is a Polish space and $d$ is a compatible complete metric. For analysts, the most familiar topology on $\mathrm{CL}(X)$ is the Hausdorff metric topology $\tau_{H}$, induced by the following infinite-valued metric on $\operatorname{CL}(X)$ :

$$
\begin{aligned}
H_{d}(A, B) & =\inf \left\{r>0: S_{r}[A] \supset B \text { and } S_{r}[B] \supset A\right\} \\
& =\sup \{|d(x, A)-d(x, B)|: x \in X\} .
\end{aligned}
$$

As is well known [CV, $\mathrm{KT}, \mathrm{Ku}], H_{d}$ is a complete metric (if the reader is bothered by infinite values, $H_{d}$ may be replaced by $\left.\min \left\{H_{d}, 1\right\}\right)$. But unless $X$ is compact, the hyperspace $\left\langle\mathrm{CL}(X), \tau_{H}\right\rangle$ fails to be separable. To see this, because $X$ cannot be totally bounded, for some $\varepsilon>0, X$ has an infinite $\varepsilon$-discrete subset $E$. Evidently, each subset of $E$ is closed, and if $A$ and $B$ are distinct nonempty subsets of $E$, we have $H_{d}(A, B) \geq \varepsilon$. A similar analysis shows that the Attouch-Wets topology, an important recent modification of the Hausdorff metric topology, fails to be separable when closed balls are not compact [ALW]. This topology seems to have become the topology of choice for hyperspaces of convex sets, in view of its stability with respect to duality in arbitrary normed linear spaces [Be4] and its overall tractability.

For topologists, the most familar topology on $\mathrm{CL}(X)$ is the Vietoris topology [KT, Mi]. This topology is, generically, a "hit-and-miss" topology, and is of a character much different than the Hausdorff metric topology. To describe this topology, we need to introduce some additional notation. Given $E \subset X$, we define these two subsets of $\operatorname{CL}(X)$ :

$$
\begin{aligned}
& E^{-} \equiv\{A \in \mathrm{CL}(X): A \cap E \neq \varnothing\}, \\
& E^{+} \equiv\{A \in \mathrm{CL}(X): A \subset E\} .
\end{aligned}
$$

Notice that $A \in E^{-}$if $A$ hits $E$, and $A \in E^{+}$if $A$ misses $E^{c}$. The Vietoris topology $\tau_{V}$ has as a subbase all sets of the form $V^{-}$where $V$ is an open subset of $X$, and $W^{+}$where $W$ is an open subset of $X$. As is evident, the finite subsets of $X$ are dense in the hyperspace, whence the finite subsets of a countable dense subset $D$ of $X$ will be dense. As a result, $\tau_{V}$ is separable. However, the Vietoris topology can be metrizable only if $X$ is compact [Ke].

A modification of the Vietoris topology, compatible with Kuratowski convergence of sequences of sets [Ku, §29] in any metric space [Be1], does produce a Polish hyperspace for locally compact Polish spaces [At, KT]: the Fell topology [Fe, Ef, Be2], also called the topology of closed convergence. Specifically, a subbase for the Fell topology $\tau_{F}$ consists of all sets of the form $V^{-}$where $V$ is open, and all sets of the form $\left(K^{c}\right)^{+}$where $K$ is compact. Without local compactness, the topology fails to be Hausdorff. 


\section{The WiJsMan topologY}

The topology that works for our purposes is the so-called Wijsman topology, studied extensively over the past twenty years by an international cast of characters [Be2, BLLN, Co, DL, FLL, He1, He2, LL, Na]. Sequential convergence in this topology was introduced by Wijsman [Wi] in the context of convex analysis. Wijsman's paper may be viewed as a point of departure for considerable subsequent research on the convergence of convex sets and functions (see, e.g., [At, Be3, Be4, BF, BP, Ts, Ma, Mo, So, SW]).

Normally, we think of the distance functional $(x, A) \rightarrow d(x, A)$ as a function of the point argument with the set held fixed. However, we may equally well regard this as a function of the set argument with the point held fixed. Keeping this in mind, we make the following definition.

Definition. Let $\langle X, d\rangle$ be a metric space. The Wijsman topology $\tau_{W}$ on $\operatorname{CL}(X)$ is the weak topology generated by $\{d(x, \cdot): x \in X\}$.

It is easy to check that $\left\langle\mathrm{CL}(X), \tau_{W}\right\rangle$ is Hausdorff, and as a weak topology, it is automatically completely regular, as the defining functions give rise to a natural compatible uniformity (see, e.g., [En, p. 537]). Now if $X$ has a countable dense subset $D$, then the following countable collection serves as a subbase for $\tau_{W}$ :

$$
\left\{d(x, \cdot)^{-1}(\alpha, \beta): x \in D, \alpha \text { rational, } \beta \text { rational }\right\} .
$$

As a result, the hyperspace is second countable provided $X$ is separable. Thus, by the Urysohn metrization theorem [Ku, p. 241], the hyperspace is metrizable and separable whenever $X$ has these properties. These facts were all observed in a slightly different way in [FLL].

There is another way to view $\tau_{W}$, more suitable for our immediate purposes. Evidently, a net $\left\langle A_{\lambda}\right\rangle$ of closed sets is $\tau_{W}$-convergent to $A \in \mathrm{CL}(X)$ if and only if for each $x \in X$, we have $d(x, A)=\lim d\left(x, A_{\lambda}\right)$. Thus, we may view $\left\langle\mathrm{CL}(X), \tau_{W}\right\rangle$ as sitting in the function space $C_{p}(X, R)$, under the identification $A \leftrightarrow d(\cdot, A)$.

In this author's view, a most significant fact about this topology was observed by Hess in [He1, $\mathrm{He} 2]$ : if $X$ is separable, then the sigma algebra generated by the Wijsman topology on $\mathrm{CL}(X)$ agrees with the Effros sigma algebra, generated by $\left\{V^{-}: V\right.$ open in $\left.X\right\}$. As a result, for separable metric target spaces, weak measurability for closed-valued multifunctions (see, e.g., [CV, KT, Hi]) can be described in a manner consistent with the usual treatment for single-valued functions.

We finally note that the Wijsman topology corresponding to each fixed metric $d$ is in general weaker than the Hausdorff metric topology $\tau_{H}$, because Hausdorff metric convergence amounts to uniform convergence of distance functionals. Each Wijsman topology is also weaker than the Vietoris topology. Remarkably, it can be shown [BLLN] that the supremum of the Wijsman topologies 
corresponding to all compatible metrics for $X$ yields the Vietoris topology! Each Wijsman topology is stronger than the Fell topology, and it follows from results of [Be2] that the infimum of the Wijsman topologies corresponding to all compatible metrics for $X$ yields the Fell topology when $X$ is locally compact. All of the topologies agree when $X$ is compact. In [BLLN], necessary and sufficient conditions for these topologies to pairwise coincide are given.

\section{4. $\left\langle\mathrm{CL}(X), \tau_{W}\right\rangle$ is Polish when $X$ is Polish}

To show that a separable metrizable space is Polish, by Alexandroff's Theorem [Ku, p. 408], it is sufficient to show that it is a $G_{\delta}$-subset of some complete metric space. We execute this for the space $\left\langle\mathrm{CL}(X), \tau_{W}\right\rangle$ in two steps. First, we show that the closure $\overline{\mathrm{CL}(X)}$ of $\mathrm{CL}(X)$ in $C_{p}(X, R)$ (under the identification $A \leftrightarrow d(\cdot, A))$ is Polish, and then we show that $\mathrm{CL}(X)$ is a $G_{\delta}$-subset of $\overline{\mathrm{CL}(X)}$.

Example. The pointwise limit of a sequence of distance functions for closed subsets of a separable complete metric space need not be a distance function. Let $X=\left\{a_{1}, a_{2}, a_{3}, \ldots\right\}$ be a countable set, equipped with the metric: $d\left(a_{1}, a_{n}\right)=2$ for $n>1$ and $d\left(a_{i}, a_{n}\right)=1$ for $1<i<n$. For each $n$, let $A_{n}=\left\{a_{1}, a_{n}, a_{n+1}, a_{n+2}, \ldots\right\}$. Then $\left\langle d\left(\cdot, A_{n}\right)\right\rangle_{1}^{\infty}$ is pointwise convergent to $f: X \rightarrow R$ defined by

$$
f\left(a_{n}\right)= \begin{cases}0 & \text { if } n=1 \\ 1 & \text { if } n \neq 1 .\end{cases}
$$

Evidently, $f$ is not a distance function of a closed set.

We start by recording some routine facts about $\overline{\mathrm{CL}(X)}$, valid without completeness or separability.

Lemma 4.1. Let $\langle X, d\rangle$ be a metric space, and let $x_{0} \in X$ be fixed. For each $f \in \overline{\mathrm{CL}(X)}$, the following statements are valid:

(a) for all $x \in X, f(x) \geq 0$;

(b) $f$ is Lipschitz with constant 1;

(c) for all $x \in X$, we have $f(x) \leq f\left(x_{0}\right)+d\left(x, x_{0}\right)$;

(d) let $A=\{x: f(x)=0\}$. Then $f=d(\cdot, A)$ if and only if $A \neq \varnothing$, and for each $x \in X$, we have $d(x, A) \leq f(x)$.

Proof. Distance functionals possess properties (a), (b), and (c), and these are preserved under pointwise convergence. To illustrate, we just prove (c), leaving (a) and (b) to the reader.

Fix $x \in X$ and let $\varepsilon>0$. Since $f \in \overline{\mathrm{CL}(X)}$, there exists $A \in \mathrm{CL}(X)$ such that $|f(x)-d(x, A)|<\varepsilon / 2$ and $\left|f\left(x_{0}\right)-d\left(x_{0}, A\right)\right|<\varepsilon / 2$. Since $d(x, A) \leq$ $d\left(x, x_{0}\right)+d\left(x_{0}, A\right)$, we obtain

$$
f(x)<d(x, A)+\varepsilon / 2<d\left(x_{0}, A\right)+d\left(x, x_{0}\right)+\varepsilon / 2<f\left(x_{0}\right)+d\left(x, x_{0}\right)+\varepsilon .
$$

This establishes (c) because $\varepsilon$ was arbitrary. 
We now turn to (d). Necessity of the conditions is obvious. For sufficiency, we need only show that $f(x) \leq d(x, A)$ for each $x$, whence $f(x)=d(x, A)$ follows from the hypothesis $d(x, A) \leq f(x)$. Fix $x \in X$. Suppose to the contrary that $d(x, A)<f(x)$ holds. Set $\varepsilon=f(x)-d(x, A)$, and pick $a \in A$ with $d(x, a)<d(x, A)+\varepsilon / 3$. Since $f \in \overline{\operatorname{CL}(X)}$, there exists $B \in \operatorname{CL}(X)$ such that $|f(a)-d(a, B)|<\varepsilon / 3$ and $|f(x)-d(x, B)|<\varepsilon / 3$. Since $f(a)=0$, we obtain this inequality string:

$$
\begin{aligned}
f(x) & <d(x, B)+\varepsilon / 3 \leq d(x, a)+d(a, B)+\varepsilon / 3 \\
& <d(x, a)+f(a)+2 \varepsilon / 3<d(x, A)+\varepsilon .
\end{aligned}
$$

This contradicts the definition of $\varepsilon$, completing the proof.

We next show that $\overline{\mathrm{CL}(X)}$, as a subspace of $C_{p}(X, R)$, is somewhat more than hemicompact [En, p. 216].

Lemma 4.2. Let $\langle X, d\rangle$ be a metric space. Then there exists an increasing sequence $\left\langle\Delta_{n}\right\rangle$ of compact subsets of $\overline{\mathrm{CL}(X)}$ such that $\overline{\mathrm{CL}(X)}=\bigcup_{n=1}^{\infty} \Delta_{n}$, and for each $n$ we have $\Delta_{n} \subset$ int $\Delta_{n+1}$.

Proof. Fix $x_{0} \in X$, and for each $n \in Z^{+}$, let $\Delta_{n}$ be this set:

$$
\Delta_{n}=\left\{f \in \overline{\mathrm{CL}(X)}: f\left(x_{0}\right) \leq n\right\} .
$$

Clearly, $\Delta_{n}$ is closed $C_{p}(X, R)$ and by, Lemma 4.1(b)-(c), $\Delta_{n}$ is both equicontinuous and pointwise bounded. Now the topology of pointwise convergence agrees with the compact open topology on $\overline{\mathrm{CL}(X)}$ by equicontinuity. Since metric spaces are $k$-spaces, by a standard version of the Ascoli Theorem (see, e.g., [MN, Theorem 3.2.6] or [En, Theorem 3.4.20]), $\Delta_{n}$ is compact in the compactopen topology and thus in the topology of pointwise convergence. Clearly, we have $\overline{\mathrm{CL}(X)}=\bigcup_{n=1}^{\infty} \Delta_{n}$ and

$$
\begin{aligned}
\Delta_{n} & \subset\left\{f \in \overline{\mathrm{CL}(X)}: f\left(x_{0}\right)<n+1\right\} \\
& =\operatorname{int}\left\{f \in \overline{\mathrm{CL}(X)}: f\left(x_{0}\right) \leq n+1\right\}=\operatorname{int} \Delta_{n+1} .
\end{aligned}
$$

Theorem 4.3. Let $X$ be a Polish space and let $d$ be a compatible complete metric for $X$. If $\tau_{W}$ is the Wijsman topology corresponding to $d$, then the hyperspace $\left\langle\mathrm{CL}(X), \tau_{W}\right\rangle$ is Polish.

Proof. By Lemma 4.2, $\overline{\mathrm{CL}(X)}$ equipped with the topology of pointwise convergence is a locally compact Hausdorff space. By equicontinuity of functions and separability of $X, \overline{\mathrm{CL}(X)}$ is easily seen to be second countable. Since locally compact spaces are completely regular, by the Urysohn metrization theorem, $\overline{\mathrm{CL}(X)}$ is second countable, metrizable, and locally compact, so that $\overline{\mathrm{CL}(X)}$ is Polish. It remains to show that $\mathrm{CL}(X)$ is a $G_{\delta}$-subset of its closure.

Let $D=\left\{x_{1}, x_{2}, x_{3}, \ldots\right\}$ be a countable dense subset of $X$. For each two positive integers $n$ and $k$, we define a subset $\Omega(n, k)$, of $\overline{\mathrm{CL}(X)}$ as follows:

$$
\begin{aligned}
& \Omega(n, k) \equiv\left\{f \in \overline{\mathrm{CL}(X)}: f^{-1}\left[0,2^{n-1}\right) \neq \varnothing\right. \text { and } \\
&\left.d\left(x_{k}, f^{-1}\left[0,2^{-n-1}\right)\right)<f\left(x_{k}\right)+2^{-n}\right\} .
\end{aligned}
$$


It is routine to check that each such set is open in $\overline{\operatorname{CL}(X)}$. We claim that

$$
\mathrm{CL}(X)=\bigcap_{n=1}^{\infty} \bigcap_{k=1}^{\infty} \Omega(n, k) .
$$

Evidently, $\mathrm{CL}(X) \subset \bigcap_{n=1}^{\infty} \bigcap_{k=1}^{\infty} \Omega(n, k)$, for if $f=d(\cdot, A)$ with $A \in \mathrm{CL}(X)$ and $k$ and $n$ are arbitrary positive integers, then

$$
\begin{aligned}
d\left(x_{k},\left\{x: d(x, A)<2^{-n-1}\right\}\right) & \leq d\left(x_{k},\{x: d(x, A)=0\}\right) \\
& =d\left(x_{k}, A\right)=f\left(x_{k}\right)<f\left(x_{k}\right)+2^{-n} .
\end{aligned}
$$

For the other inclusion, fix $f \in \bigcap_{n=1}^{\infty} \bigcap_{k=1}^{\infty} \Omega(n, k)$. By Lemma 4.1(d), it suffices to show that $A \equiv\{x: f(x)=0\}$ is nonempty, and for each $x \in X$, we have $d(x, A) \leq f(x)$. To this end, fix $p \in X$ and $\varepsilon>0$. We intend to produce $a \in A$ with $d(p, a)<f(p)+\varepsilon$.

First, choose $y_{0}=x_{k_{0}} \in D$ with $d\left(y_{0}, p\right)<\varepsilon / 8$. Since $f$ is Lipschitz with constant 1 , we have $f\left(y_{0}\right)<f(p)+\varepsilon / 8$. Choose $N \in Z^{+}$so large that $2^{-N}<$ $\varepsilon / 4$. Since $f \in \Omega\left(N, k_{0}\right)$ and $D$ is dense in the open set $f^{-1}\left[0,2^{-N-1}\right)$, there exists $y_{1} \in D$ with $f\left(y_{1}\right)<2^{-N-1}$ such that

$$
d\left(y_{1}, y_{0}\right)<f\left(y_{0}\right)+2^{-N}<f(p)+\varepsilon / 8+2^{-N} \text {. }
$$

As $y_{1} \in D$, there exists $k_{1} \in Z^{+}$with $y_{1}=x_{k_{1}}$. Since $f \in \Omega\left(N+1, k_{1}\right)$ and $D$ is dense in $f^{-1}\left[0,2^{-N-2}\right)$, there exists $y_{2} \in D$ with $f\left(y_{2}\right)<2^{-N-2}$ satisfying

$$
d\left(y_{2}, y_{1}\right)<f\left(y_{1}\right)+2^{-N-1}<2^{-N-1}+2^{-N-1}=2^{-N} \text {. }
$$

Suppose now that $\left\{y_{1}, y_{2}, \ldots, y_{n}\right\} \subset D$ have been chosen with

$$
\begin{aligned}
f\left(y_{i}\right) & <2^{-N-i}, \quad i=1,2,3, \ldots, n, \\
d\left(y_{i+1}, y_{i}\right) & <2^{-N-i+1}, \quad i=1,2,3, \ldots, n-1 .
\end{aligned}
$$

Now $y_{n}=x_{k_{n}}$ for some $k_{n} \in Z^{+}$. Since $f \in \Omega\left(N+n, k_{n}\right)$ and $D$ is dense in $f^{-1}\left[0,2^{-N-n-1}\right)$, there exists $y_{n+1} \in D$ with $f\left(y_{n+1}\right)<2^{-N-n-1}$ such that

$$
d\left(y_{n+1}, y_{n}\right)<f\left(y_{n}\right)+2^{-N-n}<2^{-N-n}+2^{-N-n}=2^{-N-n+1} .
$$

This shows that we may produce a sequence $\left\langle y_{n}\right\rangle_{1}^{\infty}$ from $D$ with $d\left(y_{n+1}, y_{n}\right)<$ $2^{-N-n+1}$ and $f\left(y_{n}\right)<2^{-N-n}$ for $n=1,2,3,4, \ldots$ By the completeness of $X,\left\langle y_{n}\right\rangle_{1}^{\infty}$ converges to some point $a \in X$, and by the continuity and nonnegativity of $f$, we have $f(a)=\lim _{n \rightarrow \infty} f\left(y_{n}\right)=0$. This shows that 
$A=\{x \in X: f(x)=0\}$ is nonempty. Finally, we have

$$
\begin{aligned}
d(p, a) & \leq d\left(p, y_{0}\right)+d\left(y_{0}, y_{1}\right)+\sum_{n=1}^{\infty} d\left(y_{n}, y_{n+1}\right) \\
& <\frac{\varepsilon}{8}+\left(f(p)+\frac{\varepsilon}{8}+2^{-N}\right)+\sum_{n=1}^{\infty} 2^{-N-n+1} \\
& <f(p)+\frac{\varepsilon}{4}+2^{-N}+2^{-N+1}<f(p)+\varepsilon .
\end{aligned}
$$

Since $d(p, A) \leq d(p, a)$ and $\varepsilon$ was arbitrary, we get $d(p, A) \leq f(p)$. We conclude by Lemma $4.1(\mathrm{~d})$ that $f=d(\cdot, A)$ so that $f \in \mathrm{CL}(X)$. This establishes the claim that $\mathrm{CL}(X)=\bigcap_{n=1}^{\infty} \bigcap_{k=1}^{\infty} \Omega(n, k)$. In summary, $\mathrm{CL}(X)$ equipped with $\tau_{W}$ is a $G_{\delta}$-subset of a Polish space and is thus Polish.

It is not hard to show that for any metric space $X$, the map $x \rightarrow\{x\}$ is embedding of $X$ into $\left\langle\mathrm{CL}(X), \tau_{W}\right\rangle$ ([Co, Proposition 2.1] or [LL, Lemma 2.7]). Moreover, the image of $X$ is closed in the hyperspace, for if $A \in \mathrm{CL}(X)$ contains two distinct points $a_{1}$ and $a_{2}$ and $r=d\left(a_{1}, a_{2}\right)$, then if $\mid d\left(a_{1}, B\right)-$ $d\left(a_{1}, A\right) \mid<r / 2$ and $\left|d\left(a_{2}, B\right)-d\left(a_{2}, A\right)\right|<r / 2$, the set $B$ cannot be a singleton. As a result, if $\left\langle\mathrm{CL}(X), \tau_{W}\right\rangle$ is completely metrizable and separable, then the underlying space must be also.

Following Wijsman [Wi], Cornet [Co], and Salinetti and Wets [SW], Levi and Lechicki [LL] adjoined the empty set to $\left\langle\mathrm{CL}(X), \tau_{W}\right\rangle$ in a general metric space $X$ by declaring a net $\left\langle A_{\lambda}\right\rangle$ convergent to $\varnothing$ provided for each $x \in X$, $\lim d\left(x, A_{\lambda}\right)=\infty$. Put differently, for a fixed $x_{0} \in X$, a (countable) local base at $\varnothing$ for the extended Wijsman topology on $\operatorname{CL}(X) \cup\{\varnothing\}$ consists of all sets of the form

$$
\{\varnothing\} \cup\left\{A \in \mathrm{CL}(X): d\left(x_{0}, A\right)>n\right\} \quad\left(n \in Z^{+}\right) .
$$

Assume now that $X$ is separable and $d$ is a complete metric. If we identify $\{\varnothing\}$ with the point at infinity in the one-point compactification $\overline{\mathrm{CL}}(X)^{*}$ of the locally compact space $\overline{\mathrm{CL}(X)}$, we have

$$
\{\varnothing\} \cup\left\{A \in \mathrm{CL}(X): d\left(x_{0}, A\right)>n\right\}=\Delta_{n}^{c} \cap(\{\varnothing\} \cup \mathrm{CL}(X)) .
$$

Since $\left\{\Delta_{n}: n \in Z^{+}\right\}$is cofinal in the compact subsets of $\overline{\operatorname{CL}(X)}$, the family $\left\{\Delta_{n}^{c}: n \in Z^{+}\right\}$forms a local base at $\varnothing$ for the one-point compactification. Thus, we see that the extended Wijsman topology on $\operatorname{CL}(X) \cup\{\varnothing\}$ is the subspace topology it inherits from the compactification. Since $\overline{\operatorname{CL}(X)}$ is second countable, so is $\overline{\mathrm{CL}(X)}{ }^{*}$, so that the compactification is completely metrizable. Since $\{\varnothing\}$ and $\operatorname{CL}(X)$ are both $G_{\delta}$-subsets of the one-point compactification, so is their union.

Combining these facts, we have established

Theorem 4.4. Let $X$ be a Polish space and let $d$ be a compatible complete metric for $X$. Then $\mathrm{CL}(X) \cup\{\varnothing\}$ equipped with the extended Wijsman topology is Polish. 
When closed and bounded subsets of $X$ are compact, it can be shown that $\mathrm{CL}(X)$ is closed in $C_{p}(X, R)$, under the identification $A \leftrightarrow d(\cdot, A)$. The extended Wijsman topology thus coincides with the one-point compactification of $\left\langle\mathrm{CL}(X), \tau_{W}\right\rangle$, as observed by Levi and Lechicki [LL].

Suppose now that $\langle X, d\rangle$, is complete but not necessarily separable. We next consider the place of the set $K(X)$ within $\left\langle\operatorname{CL}(X), \tau_{W}\right\rangle$. Evidently, $K(X)$ is not closed, for in the line $R$ with the usual metric, the sequence of intervals $\langle[-n, n]\rangle_{1}^{\infty}$ is $\tau_{W}$-convergent to $R$ itself. We intend to show $K(X)$ is of multiplicative class two in the hyperspace. For $A \in K(X)$ and $\varepsilon>0, A$ can be covered a finite number $n(A, \varepsilon)$ of open balls of radius $\varepsilon / 2$. As a result, any $\varepsilon$-discrete subset of $A$ can contain at most $n(A, \varepsilon)$ elements. For $A \in \mathrm{CL}(X)$, we denote by $M_{\varepsilon}(A)$ the supremum of the number of elements that can occur in any $\varepsilon$-discrete subset of $A$. In the literature, $\log _{2} M_{\varepsilon}(A)$ is called the $\varepsilon$-capacity of $A$ [Lo, p. 151].

Theorem 4.5. Let $\langle X, d\rangle$ be a complete metric space. Then $K(X)$ is an $F_{\sigma \delta}$ subset of $\left\langle\mathrm{CL}(X), \tau_{W}\right\rangle$.

Proof. By completeness, $A \in \mathrm{CL}(X)$ is in $K(X)$ if and only if $A$ is totally bounded, that is, if and only if for each positive integer $n$, the number $M_{1 / n}(A)$ is finite. For each $n$, let $\Theta(n, k)=\left\{A \in \mathrm{CL}(X): M_{1 / n}(A) \leq k\right\}$. It is routine to check that $\mathrm{CL}(X) \backslash \Theta(n, k)$ is $\tau_{W^{-}}$-open, and $K(X)=\bigcap_{n=1}^{\infty} \bigcup_{k=1}^{\infty} \Theta(n, k)$.

Proposition 4.6. $K\left(R^{n}\right)$ is not a $G_{\delta}$-subset of $\left\langle\mathrm{CL}\left(R^{n}\right), \tau_{W}\right\rangle$.

Proof. When the underlying space is compact, $\tau_{H}$ is compact $[\mathrm{CV}]$ and $\tau_{W}=$ $\tau_{H}$; as a result, $K\left(R^{n}\right)$ is sigma compact. Hence $K\left(R^{n}\right)^{c}$ is a $G_{\delta}$-subset of $\left\langle\mathrm{CL}\left(R^{n}\right), \tau_{W}\right\rangle$. Since the finite subsets of $X$ are dense in $\left\langle\mathrm{CL}\left(R^{n}\right), \tau_{W}\right\rangle$, so is $K\left(R^{n}\right)$. Also, $K\left(R^{n}\right)^{c}$ is dense, for if $A \in \mathrm{CL}\left(R^{n}\right)$, then $A=\tau_{W}-\lim _{n \rightarrow \infty} A \cup$ $\{x:\|x\| \geq n\}$. By Theorem 4.3 and the Baire category theorem, $K\left(R^{n}\right)$ is not a $G_{\delta}$-subset of $\left\langle\mathrm{CL}\left(R^{n}\right), \tau_{W}\right\rangle$.

The proof of Proposition 4.6 of course shows that $K\left(R^{n}\right)$ is of first category in $\left\langle\mathrm{CL}\left(R^{n}\right), \tau_{W}\right\rangle$.

Corollary 4.7. $\left\langle K\left(R^{n}\right), \tau_{W}\right\rangle$ is not completely metrizable.

\section{ON BOREL MULTIFUNCTIONS}

Let $M$ and $X$ be metric spaces. It is standard to call a function $f: M \rightarrow$ $X$ Borel measurable (resp. Borel measurable of class $\alpha$ ) provided the inverse image of each open subset of $X$ is Borel (resp. of additive class $\alpha$ ) [Ku, $\S 30-$ 31]. Absent an agreed upon topology for $\operatorname{CL}(X)$, it has been traditional to call a closed-valued multifunction from $M$ to $X$ (see, e.g., [CV, KT]) Borel measurable (resp. Borel measurable of class $\alpha$ ) provided

$$
f^{-}(V) \equiv\{m \in M: f(m) \cap V \neq \varnothing\}
$$


is Borel (resp. of additive class $\alpha$ ) for each open subset $V$ of $X$. Perhaps the most famous result in this area is the celebrated selection theorem of Kuratowski and Ryll-Nardzewski [KR].

We may regard a multifunction $f: M \rightrightarrows X$ as a single-valued function $\hat{f}$ from $M$ to $\operatorname{CL}(X)$. When $X$ is separable, and $\operatorname{CL}(X)$ is equipped with a Wijsman topology determined by a compatible metric, it follows from a theorem of Hess [He2] that $f$ is a Borel measurable multifunction if and only if $\hat{f}: M \rightarrow$ $\left\langle\mathrm{CL}(X), \tau_{W}\right\rangle$ is a Borel measurable single-valued function. With respect to Borel measurable functions of class $\alpha$, we have

Theorem 5.1. Let $\langle M, \rho\rangle$ and $\langle X, d\rangle$ be metric spaces with $X$ separable. Let $f: M \rightrightarrows X$ be a closed-valued multifunction, and let $\hat{f}: M \rightarrow\left\langle\operatorname{CL}(X), \tau_{W}\right\rangle$ be the associated single-valued function. Then

(a) if $\hat{f}$ is of Borel class $\alpha$, then $f$ is of Borel class $\alpha$;

(b) if $f$ is of Borel class $\alpha$, then $\hat{f}$ is of Borel class $\alpha+1$.

Proof. Fix $x \in X$ and $\beta>0$. We have

$$
\hat{f}^{-1}(\{A: d(x, A)<\beta\})=f^{-}\left(S_{\beta}[x]\right) .
$$

Since $\{A: d(x, A)<\beta\}$ is $\tau_{W}$-open, each open subset of $X$ is a countable union of open balls, and the operation $f^{-}$preserves unions, we see that (a) holds.

For (b), observe that for $\alpha \geq 0$, we have

$$
\hat{f}^{-1}(\{A: d(x, A)>\alpha\})=\bigcup_{n=1}^{\infty}\left[f^{-}\left(S_{\alpha+1 / n}[x]\right)\right]^{c} .
$$

By assumption, for each $n$, the set $\left[f^{-}\left(S_{\alpha+1 / n}[x]\right)\right]^{c}$ is of multiplicative class $\alpha$ and therefore is, of additive class $\alpha+1$. From (*) and (**) we conclude that the inverse image under $\hat{f}$ of each set in a subbase for $\tau_{W}$ is of additive class $\alpha+1$. Since the additive classes are closed under finite intersections and countable unions, and the hyperspace is second countable, $\hat{f}^{-1}(\Delta)$ is of additive class $\alpha+1$ for each $\Delta \in \tau_{W}$.

The multifunction $f: R \rightrightarrows R$ defined by $f(0)=\{0\}$, and $f(x)=R$ for $x \neq 0$, is obviously of class zero, whereas $\hat{f}$ is not of class zero, that is, $\hat{f}$ is not continuous, because $\hat{f}^{-1}(\{A: d(2, A)>1\})=\{0\}$. Thus, $\alpha+1$ cannot be replaced by $\alpha$ in the statement of Theorem 5.1 .

\section{REFERENCES}

[At] H. Attouch, Variational convergence for functions and operators, Pitman, New York, 1984. [ALW] H. Attouch, R. Lucchetti, and R. Wets, The topology of the $\rho$-Hausdorff distance, Ann. Mat. Pura Appl. (to appear).

[BP] M. Baronti and P. Papini, Convergence of sequences of sets, Methods of Functional Analysis in Approximation Theory, Internat. Ser. Numer. Math., vol. 76, Birkhäuser-Verlag, Basel, 1986. 
[Be1] G. Beer, Metric spaces with nice closed balls and distance functions for closed sets, Bull. Austral. Math. Soc. 35 (1987), 81-96.

[Be2] _ _ An embedding theorem for the Fell topology, Michigan Math. J. 35 (1988), 3-9.

[Be3] _ Support and distance functionals for convex sets, Numer. Funct. Anal. Optim. 10 (1989), 15-36.

[Be4] Conjugate convex functions and the epi-distance topology, Proc. Amer. Math. Soc. 108 (1990), 117-126.

[BLLN] G. Beer, A. Lechicki, S. Levi, and S. Naimpally, Distance functionals and the suprema of hyperspace topologies, Ann. Mat. Pura Appl. (to appear).

[BF] J. Borwein and S. Fitzpatrick, Mosco convergence and the Kadec property, Proc. Amer. Math. Soc. 106 (1989), 843-849.

[CV] C. Castaing and M. Valadier, Convex analysis and measurable multifunctions, Lecture Notes in Math., vol. 580, Springer-Verlag, Berlin, 1977.

[Ch] J. P. R. Christensen, Topology and Borel structure, North-Holland, Amsterdam, 1974.

[Co] B. Cornet, Topologies sur les fermés d'un espace métrique, Cahiers de mathématiques de la décision \#7309, Université de Paris Dauphine, 1973.

[DL] I. Del Prete and M. B. Lignola, On convergence of closed-valued multifunctions, Boll. Un. Mat. Ital. B-6 (1983),819-834.

[Ef] E. Effros, Convergence of closed subsets in a topological space, Proc. Amer. Math. Soc. 16 (1965), 929-931.

[En] R. Engelking, General topology, Polish Scientific, Warsaw, 1977.

[Fe] J. Fell, A Hausdorff topology for the closed subsets of a locally compact non-Hausdorff space, Proc. Amer. Math. Soc. 13 (1962), 472-476.

[FLL] S. Francaviglia, A. Lechicki, and S. Levi, Quasi-uniformization of hyperspaces and convergence of nets of semicontinuous multifunctions, J. Math. Anal. Appl. 112 (1985), 347-370.

[He1] C. Hess, Loi de probabilité des ensembles aléatoires à valeurs fermées dans un espace metrique séparable, C. R. Acad. Sci. Paris, Series I, 296 (1983), 883-886.

[He2] __ Contributions à l'ètude de la mesurabilité, de la loi de probabilité, et de la convergence des multifunctions, Thèse d'état, Montpellier, 1986.

[Hi] C. Himmelberg, Measurable relations, Fund. Math. 87 (1975), 53-72.

[Ke] J. Keesling, Normality and properties related to compactness in hyperspaces, Proc. Amer. Math. Soc. 24 (1970), 760-766.

[KT] E. Klein and A. Thompson, Theory of correspondences, Wiley, New York, 1984.

[Ku] K. Kuratowski, Topology, vol. 1, Academic Press, New York, 1966.

[KR] K. Kuratowski and C. Ryll-Nardzewski, A general theorem on selectors, Bull. Acad. Polon. Sci. 13 (1965), 397-402.

[LL] A. Lechicki and S. Levi, Wijsman convergence in the hyperspace of a metric space, Bull. Un. Mat. Ital. 5-B (1987), 435-452.

[Lo] G. G. Lorentz, The approximation of functions, Holt, Rinehart, and Winston, New York, 1966.

[Ma] M. Matzeu, Su un tipo di continuita dell' operatore subdifferenziale, Boll. Un. Mat. Ital. (5) 14-B (1977), 480-490.

[MN] R. McCoy and I. Ntantu, Topological properties of spaces of continuous functions, Lecture Notes in Math., vol. 1315, Springer-Verlag, Berlin, 1988.

[Mi] E. Michael, Topologies on spaces of subsets, Trans. Amer. Math. Soc. 71 (1951), 152-182.

[Mo] U. Mosco, Convergence of convex sets and of solutions of variational inequalities, Adv. in Math. 3 (1969), 510-585.

[Na] S. Naimpally, Wijsman convergence for function spaces, Rend. Circ. Palermo II-18 (1988), 343-358.

[Pa] K. Parthasarathy, Probability measures on metric spaces, Academic Press, New York, 1967. 
[SW] G. Salinetti and R. Wets, On the convergence of sequences of convex sets in finite dimensions, SIAM Review 21 (1979), 18-33.

[So] Y. Sonntag, Convergence au sens de Mosco; theorie et applications à l'approximation des solutions d'inéquations, Thèse d'Etat. Université de Provence, Marseille, 1982.

[Ts] M. Tsukada, Convergence of best approximations in a smooth Banach space, J. Approx. Theory 40 (1984), 301-309.

[Wa] D. Wagner, Survey of measurable selections, SIAM J. Control. Optim. 15 (1977), 859-903.

[Wi] R. Wijsman, Convergence of sequences of convex sets, cones, and functions, II, Trans. Amer. Math. Soc. 123 (1966), 32-45.

Department of Mathematics, California State University at los Angeles, Los AngeLes, CAlifornia 90032 